\title{
Articles
}

The Person and the Challenges

Volume 6 (2016) Number 1, p. 5-16

DOI: http://dx.doi.org/10.15633/pch.1653

Wojciech Zyzak

The Ponifical University of John Paul II in Krakow, Poland

\section{Der katholische Glaubensbegriff und Toleranz. Inspirationen von Paweł Włodkowic}

\section{The Catholic Concepts of the Faith and Tolerance: Inspirations of Paweł Włodkowic}

\begin{abstract}
Paweł Włodkowic - Latin Paulus Vladimiri (1370-1435): Catholic priest, scholar, jurist, religious and political writer, rector of the Jagiellonian University, defender of Polish interests in disputes with Knights of the Teutonic Order. Paweł Włodkowic was an early teacher of the idea of tolerance, who during the Council of Constance stated that pagan nations have the right to maintain their religious beliefs. Based on the major theological authority of that time, i.e. Saint Thomas Aquinas, Włodkowic could, in the very concept of faith in the Catholic Church, see the most important elements requiring tolerance. The point is, above all, that faith must be an act of human freedom, that it is God's grace and that it is closely related to reason. These elements have been commented on by authorities such as Edith Stein and Joseph Ratzinger.
\end{abstract}

Keywords

Tolerance, faith, Włodkowic. 


\section{Einleitung}

Der bekannte katholische Schriftsteller Gilbert Keith Chesterton betonte den positiven Aspekt der spezifisch verstandenen „Intoleranz” der Kirche. Seiner Meinung nach war sie aus der Erde aufgestiegen, um Himmel und Erde des Heidentums zu zerstören. Es ging ihm genau um die Idee des Monotheismus und ausführlicher um eine Einstellung, die nach Wahrheit strebt ${ }^{1}$. Zu diesem Thema äußert sich Joseph Ratzinger detaillierter in seinem Buch „Demokratie Pluralismus - Christentum“. Ratzinger meint, der christliche Glaube habe die Idee der altertümlichen Toleranz, wegen der Unmöglichkeit der Unterordnung unter das polytheistische Pantheon, zersprengt. Der Monotheismus konnte sich nicht ausschließlich in die Privatsphäre zurückziehen, ohne auf den Anspruch nach Wahrheit zu verzichten. Deswegen musste auch das Christentum von Anfang an zumindest das Recht auf die Ablehnung der herrschenden öffentlichen Ordnung in ihrem religiösen Charakter erkämpfen. In diesem Sinne besaß das Christentum schon seit den Anfängen einen öffentlich-rechtlichen Anspruch, sich auf eine ähnliche Ebene wie der Staat zu stellen. Darauf beruht einerseits seine Größe als Ausgleich zum Totalitarismus, andererseits aber die Gefahr einer theokratischen Ausdehnung. Daher kann sich also der Anspruch auf Wahrheit im Christentum leider in eine politische Intoleranz verwandeln ${ }^{2}$. Aus der Geschichte kennen wir allzu gut Beispiele der Intoleranz, sogar der Verfolgungen im Namen des Evangeliums, zum Glück sind wir aber auch stolz auf den Geist der Toleranz, der unter anderem von dem polnischen Gelehrten, Rektor der Jagiellonen-Universität und Teilnehmer des Konzils in Konstanz Paweł Włodkowic - ausgearbeitet wurde ${ }^{3}$.

1 Vgl. G. K. Chesterton, Wiekuisty Człowiek, Warszawa 2013, S. 284.

${ }^{2}$ Vgl. J. Ratzinger, Demokratie - Pluralismus - Christentum, Leutesdorf 1984, S. $20 \mathrm{ff}$.

${ }^{3}$ Paweł Włodkowic geb. Zwischen 1370-1373 in Brudzeń Duży, gest. Ende 1435 in Krakau - katholischer Priester, Gelehrter, Jurist, religiöser und rechtlich-politischer Schriftsteller, Rektor der Jagiellonen-Universität, Verfechter der polnischen Staatsinteressen im Streit mit den Kreuzrittern. Paweł Włodkowic war ein früher Verkünder der Idee der Toleranz, der während des Konzils in Konstanz behauptete, die heidnischen Völker haben das Recht auf die Erhaltung des eigenen Glaubens.

„Postquam infideles inter Christianos volunt vivere pacifice nulla molestia in personis et rebus est eis inferenda. (...) Nec papa debet eis bona auferre, quin ymmo debet eos quam diu potest eos tollerare. (...) Et Iudei maxime tollerandi sunt“. Paweł Włodkowic, Saeventibus (1415), in: L. Ehrlich, Pisma wybrane Pawła Włodkowica, Band 1, Instytut Wydawniczy PAX, Warszawa 1968, S. 9. 


\section{Toleranz und Glaube in der Auffassung von Paweł Włodkowic}

Plus ratio quam vis, „mehr mit Verstand als mit Kraft”, das heutige Motto der Jagiellonen-Universität, fasste ursprünglich das Programm einer friedlichen und gewaltlosen Christianisierung Litauens durch die polnische Krone zusammen. Paweł Włodkowic war ein großer Verfechter dieses Prinzips auf dem europäischen Forum. Auf dem Konzil in Konstanz verteidigte er den polnischen König vor den Vorwürfen von Falkenberg. Laut Włodkowic seien die Kreuzritter die richtigen Ketzer, denn ihre These von der Bekehrung der Ungläubigen durch Gewalt bedeute Ketzerei. Was heißt das? Wie konnte er sich auf diese Weise äußern? Włodkowic predigte die Idee der Toleranz gegenüber Andersgläubigen, besonders die Achtung der Juden. Die Grundlage seiner Gedanken bildete der christliche und realistische Menschenbegriff, der die übernatürliche Würde sowie die natürliche Gleichheit und Freiheit, die allen Menschen zustehen, betonte ${ }^{4}$. Er predigte auch die Überzeugung von dem unveräußerlichen Recht jedes Menschen auf Gewissensfreiheit. Jedoch konnte Włodkowic meiner Meinung nach schon in der Glaubenswirklichkeit selbst die Quelle der Toleranz sehen, wie sie sich aus seiner berühmten Feststellung ergibt - der Glaube sei eine theologische Tugend, deswegen gehöre die Verbreitung des Glaubens zu den Theologen, nicht zu den Kämpfern ${ }^{5}$. Was ist also der Glaube, was rechtfertigt in ihm selbst, dass er von Theologen verbreitet wird und nicht von Kämpfern erzwungen wird?

Wir wissen, dass Włodkowic sich in den theologischen Schlüsselfragen an die größte Autorität von damals, nämlich an Thomas von Aquin anlehnte ${ }^{6}$.

„Preterea ex quo infideles non sunt ad fidem cogendi sed tolerandi et sanctis exhortationibus inducendi“. Paweł Włodkowic, Ad aperiendam (1416), in: L. Ehrlich, Pisma wybrane Pawła Włodkowica, Band 2, Instytut Wydawniczy PAX, Warszawa 1966, S. 79.

4 „Non est licitum infideles armis vel oppressionibus ad fidem compellere Christianam”. Paweł Włodkowic, Opinio Ostiensis (1415), in: L. Ehrlich, Pisma wybrane Pawła Włodkowica, Band 1, Instytut Wydawniczy PAX, Warszawa 1968, S. 129.

${ }^{5}$ Vgl. S. Wielgus, Paweł Włodkowic, in: Encyklopedia filozofii polskiej, Band 2, Lublin 2011, S. 306-311.

„Tales enim non asperitatibus nec vi sed blandimentis sunt ad fidem inducendi”. Paweł Włodkowic, Ad videndum (1421), in: L. Ehrlich, Pisma wybrane Pawła Włodkowica, Band 3, Instytut Wydawniczy PAX, Warszawa 1969, S. 155.

${ }^{6}$ Vgl. L. Ehrlich, Pisma wybrane Pawła Włodkowica, Band 3, Instytut Wydawniczy PAX, Warszawa 1969, S. 242-243. 
Mit Sicherheit machte er sich den Gedanken von Aquinat zunutze, der meinte, das göttliche Recht widerspreche nie dem natürlichen Recht und hebt es nicht auf, sondern gründet auf ihm. Man kann vermuten, dass er sich selbst in der Auffassung des Glaubensbegriffs auf seine Autorität stützte ${ }^{7}$. Es lohnt sich also zu sehen, wie der heilige Thomas den Glauben versteht und in welchen Punkten des christlichen Glaubensbegriffes die Möglichkeit oder selbst die Notwendigkeit dessen enthalten ist, was wir heute Toleranz nennen.

\section{Der heilige Thomas von Aquin über den Glauben}

Am ausführlichsten und zugleich sehr synthetisch äußerte sich Thomas zu den Glaubensfragen in Quaestiones disputatae De veritate q. 14. Nach dem heiligen Augustinus bezeichnete Aquinat den Glauben als ein mit der Zustimmung verbundenes Denken. Diese Bezeichnung bildet eine gemeinsame Eigenschaft des Glaubens und des Wissens. Während jedoch beim Wissen die Sicherheit durch das Denken determiniert wird, stehen im Glaubensakt der Gedanke und die Zustimmung im Gleichgewicht, ex aequo. Im Glauben erscheint die Zustimmung nicht als eine ersichtliche Klarheit, sondern sie wird durch den Willensakt verursacht. Der Wille geht in bestimmter Weise der Erkenntnis voraus und bewirkt, dass wir dem Glaubensinhalt dank der Berührung durch Gott zustimmen. Dank dieser Berührung durch Gott weiß der Glaube, dass das Wahrheit ist, was für den Verstand unklar erscheint. Kardinal Ratzinger schrieb im Buch „Weggemeinschaft des Glaubens“, indem er die „Mechanismen“ der Wirkung des Glaubens betrachtete, dass seine Folge die Freiwilligkeit des Glaubensaktes sei, denn glauben könne man nur freiwillig. Wie Ratzinger schreibt, behandele Thomas den Glauben zuerst als einen theoretischen Akt im Unterschied zu anderen theoretischen Akten. Gemeinsam ist, dass der Glaube sich auf die Wahrheit, als seinen Gegenstand, richtet. Er gehört also wesentlich zu der Erkenntnisgattung. Mit dem Verstehen und Wissen teilt er die Sicherheit

7 „Unde beatus doctor (2a 2e questione X articulo VIII) dicit quod gentiles et Iudei et tales qui fidem non susceperunt nullo modo sunt ad fidem compellendi ut ipsi credant, quia credere voluntatis est et propter hoc fideles Christi frequenter contra infideles bellum movent non quidem ut eos ad credendum cogant, quia si eos eciam vicissent et captivos haberent in eorum libertate relinquerent an credere velent, sed propter hoc ut eos compellant ne fidem impediant". Paweł Włodkowic, Ad aperiendam (1416), in: L. Ehrlich, Pisma wybrane Pawła Włodkowica, Band 2, Instytut Wydawniczy PAX, Warszawa 1966, S. 45. 
und Bestätigung der aufgefassten Wahrheit und unterscheidet sich dadurch vom Zweifel und von Meinungen, denen es an Sicherheit fehlt. Vom Verstehen und Wissen trennt ihn die Tatsache, dass die Bestätigung nicht auf einer direkten Betrachtung oder logischer Folgerung der aufgefassten Wahrheit beruht, sondern dass das Motiv des Willens den Verstand zu Bestätigung veranlasst. Um jedoch mit dem Glauben zu tun zu haben, muss der Wille freiwillig und unter dem Einfluss der Gnade Gottes wirken ${ }^{8}$.

An dieser Stelle sehen wir, warum der Glaube vom Hörensagen und nicht vom Verstehen kommt. Das Denken im Glaubensakt wird nicht beendet und findet darin keine Ruhe. Der Glaube ist eine Wallfahrt des Denkens, worin der Verstand in der Suchspannung bleibt. Im Glaubensakt wird der Verstand „von außen bis zum Ende geführt, d.h. in bestimmter Weise gefangengenommen”. Deswegen, trotz der starken Willensentscheidung, bleibt immer noch die umgekehrte Bewegung (motus de contrario). Das Denken muss sich bemühen, die Willensentscheidung zu erreichen und motus de contrario zu überwinden. Darauf beruht die Rolle der Theologie als Wissenschaft, denn der Glaube sucht nach Verständnis. Deswegen auch kann man im engeren Sinne keinen Menschen mit Argumenten vom Glauben überzeugen, man kann jedoch zeigen, dass der Glaube nicht irrational ist.

In „De veritate“ unterschied Thomas den Glauben als eine Tugend (fides, qua creditur) davon, woran wir glauben, d.h. von der offenbarten Wahrheit (fides, quae creditur) und endlich von der lebendigen Aktivierung dieser Tugend, d.h. dem Glaubensakt (credere). Im lebendigen Glauben führte er die Annahme der Wahrheiten des Glaubens in Anbetracht der Autorität Gottes an, also „dem Gott glauben (credere Deo)“, was den Glauben an Gott voraussetzt (credere Deum) und „sich an Gott im Glauben wenden“, d.h. nach ihm streben, "Glauben an Gott“, was Thomas credere in Deum nannte. Das zeigt, der Glaube ist nicht nur ein intellektueller Akt, sondern auch ein Akt des ganzen Menschen, der sich Gott anvertraut. Zu einer solchen Haltung kann man niemanden zwingen.

Dem Glaubensakt, in der Lehre des Heiligen Thomas, entspricht der Habitus, als eine ständige Einstellung des Verstandes auf die unsichtbare Wahrheit und einfache Zustimmungsbereitschaft mit ihr im Glaubensakt. Da der Wille dem Verstand diese feste Richtung angibt, sollten wir im Habitus des Glaubens eine

${ }^{8}$ Vgl. Thomas von Aquin, Quaestiones disputatae "De veritate", q. 14; vgl. J. Ratzinger, Weggemeinschaft des Glaubens, Augsburg 2002, S. 18-21. 
Tugend sehen. Um folgendes zu erreichen, sollte der Glaube durch die Liebe vervollkommnet werden. Der Glaube, der den Namen einer Tugend verdient, sollte durch die Liebe geformt werden. Da der Glaube dem Verstand die Kraft gibt, die ihm vorgelegene Wahrheiten aufzufassen, ist er eine „eingegossene“ Tugend, d.h. Gottes Gabe, die man - was wiederholt betont werden sollte nicht erzwingen kann. Das Leben des Glaubens ist ein ständiges, gegenseitiges Eingreifen des Verstandes und des Willens, der Gnade und der Freiheit?.

Im folgenden Teil dieser Reflexion sollten wir ausführlicher die im Glaubensakt enthaltene Dimension der Freiheit und der Gnade betrachten und die Aufmerksamkeit auf das Verhältnis des Glaubens zum Verstand richten. Gerade in diesen Grundeigenschaften des Glaubens ist die Grundlage der Toleranzidee enthalten.

\section{Die Dimension der Freiheit im Glauben}

Der heilige Johannes Paul II. äußerte sich mehrmals zum Thema der Evangelisierung und knüpfte dabei an die Toleranzidee von Paweł Włodkowic an. Im Jahre 1979 in Auschwitz-Birkenau sprach er: „Ich möchte zurückkommen auf die Weisheit des Altmeisters Paweł Włodkowic, des Rektors der Jagiellonen-Universität von Krakow (Krakau), der gelehrt hat, dass folgende Rechte der Nationen zu sichern seien: ihre Rechte auf Existenz, auf Freiheit, auf Unabhängigkeit, auf eine eigene Kultur und auf eine würdige Entwicklung. Włodkowic schreibt: Wo die Macht stärker wirkt als die Liebe, sucht man die eigenen Interessen und nicht Jesus Christus; folglich entfernt man sich leicht von der Norm des göttlichen Gesetzes. Jede Art von Recht verurteilt den, der Menschen überfällt, die in Frieden leben wollen: das Naturrecht nach dem Prinzip: Was du für dich selbst willst, das tu auch dem anderen, und das göttliche Recht, denn im Gebot: Du sollst nicht stehlen, wird jede Art von Raub verboten und im Gebot: Du sollst nicht töten, jegliche Gewalt"10. Die vom Papst betonte Logik von Włodkowic ist einfach. Der Gläubige verkündet Christus, weil er sein größtes Gut und seine größte Freude ist,

9 Vgl. J. Ratzinger, Weggemeinschaft des Glaubens, Augsburg 2002, S. 22-25.

${ }^{10}$ Vgl. Johannes Paul II, Predigt von Papst Johannes Paul II am 7. Juni 1979 in Auschwitz-Birkenau. Vgl. Paweł Włodkowic, Saeventibus (1415), in: L. Ehrlich, Pisma wybrane Pawła Włodkowica, Band 1, Instytut Wydawniczy PAX, Warszawa 1968, S. 58-61. 
und er möchte ihn mit anderen Menschen teilen. Aber als Wirkungsmethode der Kirche gilt das Respektieren menschlicher Freiheit. Die Kraft ist eine ideologische Waffe, und sie tötet den Glauben. Das Evangelium dagegen ist eine Botschaft der Liebe und wendet sich an den freien Menschen. Die Freiheit ist eine notwendige Bedingung, um Christus anzunehmen. Hier sieht man den Einfluss der Gedanken von Włodkowic - die einzige Sache, die von der Toleranz ausgeschlossen wird, ist das Benutzen von Methoden, die im Widerspruch mit dem Respektieren des freien Aktes stehen, d.h. mit der Wahrheitserkenntnis, und die beabsichtigen würden, den anderen die Wahrheit mit Gewalt aufzudrängen.

Übrigens, wenn der Glaube eine Tugend ist, muss er ein freier Akt sein, denn nur dann kann er aus dem ethischen Gesichtspunkt ein verdienstlicher Akt werden. Diese Tatsache können die Vernunftargumente auch nicht ändern und vom Glauben kann man niemanden überzeugen. Natürlich sprechen das Alte und das Neue Testament über die Möglichkeit, das Existieren des Schöpfers an seinen Geschöpfen zu erkennen (vgl. Weish 13 und Gen 1). Das Erste Vatikanische Konzil erhob diese Wahrheit sogar zum Dogma. In der Philosophie spricht man traditionell über die Wege der Gotteserkenntnis, und heute eher nicht über die Beweise, dass Gott existiert. Der heilige Thomas unterschied jedoch schon die Vernunfterkenntnis der Existenz Gottes aus den Geschöpfen vom lebendigen Akt des Glaubens an ihn. Edith Stein, die den Weg vom Atheismus zum Christentum ging, betonte, dass die Vernunftargumente nützlich sein können, aber letztendlich muss der Mensch selbst eine Entscheidung in Freiheit treffen, die Entscheidung, die sie mit einem Sprung über den Abgrund verglich $^{11}$.

\section{Die Dimension der Gnade im Glauben}

Die früheren Betrachtungen zeigten, dass der Glaubensakt ein Freiheitsakt unter dem Einfluss der Gnade Gottes ist. Hier berühren wir schon das Geheimnis. Włodkowic schrieb, dass obwohl die Andersgläubigen nicht vom Schafstall der Kirche kommen, sie alle jedoch ohne Zweifel Christi Schafe sind, der Schöpfung zufolge. Christus sprach nämlich, er habe andere Schafe, die

${ }^{11}$ Vgl. E. Stein, Endliches und ewiges Sein. Versuch eines Aufstiegs zum Sinn des Seins, Freiburg im Breisgau 1986, s. 105 f. 
nicht aus diesem Schafstall sind ${ }^{12}$. Warum gibt es nicht einen Schafstall? Ist die Freiheit des Menschen die einzige Antwort? Edith Stein, gewiss aufgrund eigener Erfahrungen, war bereit, die hartherzigen Atheisten von denjenigen Menschen zu unterscheiden, die erst nach Wahrheit suchen, „noch nicht glauben“, denn die Stunde ist noch nicht gekommen (die Stunde der Gnade)! ${ }^{13}$.

Der Anteil der Freiheit und der Gnade im Glaubensakt beruht darauf, dass einerseits der Glaube eine Tugend ist und dass wir dank ihrer Kraft in Freiheit die offenbarte Wahrheit annehmen. Andererseits ist die Zustimmung für die offenbarte Wahrheit nicht blind, denn sie beruht auf der Erleuchtung und Beseelung durch den Heiligen Geist. Deswegen wirken auch die Gnade und die Freiheit im Glaubensakt zusammen. Die Hilfe der Gnade, die den Glauben ermöglicht, beruht auf dem Darbieten der Wahrheit und auf der Stärkung des Verstandes, um sie aufzufassen, aber auch auf dem Erteilen einer festen Willenshaltung. Da jedoch der Glaube sich nicht mit der Erkenntnis und Liebe der Erlösten im Himmel messen kann, ist die Entscheidung im Glauben nicht so offensichtlich und fehlerfrei wie im Himmel und kann von anderen Verstandesund Willensmotiven beiseite geschoben werden. Deswegen besteht zu Lebzeiten für den Menschen immer die Möglichkeit sich vom Glauben zu lösen und zum Unglauben überzutreten.

Die Wahrheit über die Notwendigkeit der Wirkung der Gnade Gottes im Glauben äußerte Edith Stein ausdrücklich, indem sie schrieb, dass es unmöglich werde, ohne den Empfang der Gnade gläubig zu sein. Nur die Bereitschaft zum Glauben kann man dank der Kraft der Freiheit erreichen. Da das natürliche Licht des Verstandes nicht ausreichend ist für den Glauben und der Glaube eine Gabe der Gnade ist, erscheint die Absicht jemanden zum Glauben zu zwingen als absurd. Andererseits sind die natürlichen Motive des Verstandes und die übernatürliche Erleuchtung nicht bindend, es bleibt demnach eine Auswahlmöglichkeit. Daraus folgt, dass der Glaube eine Tat des Gehorsams in Freiheit ist. Deswegen ist er eine verdienstliche Tugend, aber man kann ihn nicht erzwingen ${ }^{14}$.

${ }^{12}$ Vgl. Paweł Włodkowic, Quoniam error (1417), in: L. Ehrlich, Pisma wybrane Pawła Włodkowica, Band 2, Instytut Wydawniczy PAX, Warszawa 1966, S. 328.

${ }^{13}$ Vgl. E. Stein, Kreuzeswissenschaft. Studie über Johannes vom Kreuz, Freiburg im Breisgau 2003, S. 153f.

${ }^{14}$ Vgl. E. Stein, Was ist der Mensch? Eine theologische Anthropologie, Freiburg im Breisgau 1994, S. 182. 


\section{Der Glaube im Verhältnis zum Verstand}

Die oben angeführte Regel „Plus ratio quam vis” setzt, indem sie die Gewalt ausschließt, eher auf den Verstand, auf die Kraft der Argumente als auf die Argumente der Kraft. Für den Gläubigen ist auch das Verhältnis zwischen dem Glauben und dem Verstand sehr eng und notwendig. Seit dem Altertum wird es historisch und konkret durch das Verhältnis des christlichen Erbes zu den Errungenschaften der Philosophie und der griechischen Kultur ausgedrückt. Zwar wurde es später aufgelockert, nämlich im Voluntarismus von Duns Scotus, aber die klassische und Włodkowic sehr nahe Richtung des Denkens des hl. Thomas war durch das Vertrauen zum Verstand gekennzeichnet. In der dominikanischen Schule knüpfte man stärker an die neu entdeckten Errungenschaften von Aristoteles an und betonte den Intellektualismus, entwickelte das Interesse an der Natur und an den äußeren und wissenschaftlichen Erfahrungen. Kardinal Joseph Ratzinger betrachtet dieses Verhältnis zwischen dem Glauben und dem Verstand als wesentlich für die Religion. Seiner Meinung nach entschied sich der christliche Glaube gegen die Götter der Religion und für den Gott der Philosophen, d.h. gegen den Gewohnheitsmythos und für die Wahrheit des Daseins selbst. Indem sich das Christentum für den Gott der Philosophen entschied und ihn als Gott verkündete, den man anbeten kann und der zum Menschen spricht, wurde er aus dem ausschließlich akademischen Kontext losgerissen. Dieser Gott erwies sich für den Glauben als der Menschen Gott, als Gott der nicht nur eine ewige Mathematik des Weltalls ist, sondern Agape, die schöpferische Macht der Liebe. Das Gleichsetzen vom Gott des Glaubens und vom Gott der Philosophen bedeutet jedoch nicht das Gleichsetzen des Glaubens und der Philosophie ${ }^{15}$. Sie sind zwei verschiedene Gebiete mit anderen Methodologien. Deswegen bestehen auch Möglichkeit und Notwendigkeit des Dialogs auf dem Weg der Wahrheitserforschung. Der Gläubige besitzt zwei Flügel (Fides et ratio), woran der hl. Johannes Paul II. erinnerte. Und wie wir glauben, sollte zwischen dem Verstand und dem Glauben kein wirklicher Widerspruch bestehen, denn ihre Quelle ist derselbe Gott. Falls es einen scheinbaren Widerspruch geben sollte, hieße das, der Glaube wurde falsch verstanden oder die wissenschaftliche Hypothese wurde unüberlegt als Wahrheit anerkannt. In diesem Kontext schrieb Edith Stein: Wenn es für alle, die nach

\footnotetext{
${ }^{15}$ Vgl. J. Ratzinger, Einführung in das Christentum. Vorlesungen über das Apostolische Glaubensbekenntnis, München 1968, S. 105-109.
} 
Wahrheit suchen, einen gewissen gemeinsamen Weg und ein gemeinsames Arbeitsgebiet gäbe, dann seien das natürliche Wissen und der Glaube, die Philosophie und Theologie nicht getrennt, als ob sie sich überhaupt füreinander nicht interessieren würden. Sie sollten im ständigen Dialog stehen ${ }^{16}$. Auf diese Weise vermeiden wir die Gefahr des Fideismus (credo quia absurdum), der zur extremen Trennung des Glaubens vom Verstand, und letztendlich oft zur Gewalt im Namen der Religion führt. Der Glaube als Annahme der Wahrheit Gottes, in Jesus Christus offenbart, ist weder für den Verstand noch für die Freiheit eine Gefahr. Der Glaube verteidigt den Verstand, weil er selbst den suchenden Menschen (fides quaerens intellectum) braucht. Ein Hindernis im Glauben ist nicht die fragende Haltung, sondern eine Haltung für welche die Wahrheit etwas Unerreichbares bedeutet. Der Glaube versucht seine Grundlage und seinen Inhalt $\mathrm{zu}$ verstehen, und wenn es in einer geordneten, methodischen Form geschieht, sprechen wir von Theologie. Gerade deswegen konnten im Mittelalter Universitäten entstehen, da der Glaube erkannte, dass die Suche nach Wahrheit möglich ist. Demnach ist die Universität das Produkt einer Aufgabe, die dem Verstand aufgelegt wurde und sich im christlichen Glaubensakt befindet ${ }^{17}$.

\section{Schlussfolgerungen}

Aus der Geschichte wissen wir, dass der Versuch, den Glauben durch moderne Wissenschaften oder mithilfe staatlicher Unterstützung zu verteidigen, gescheitert ist, ihn innerlich leerte und an seiner geistigen Regeneration hinderte. Das Verwechseln des Glaubens an die absolute in Christus offenbarte Wahrheit mit dem irdischen rechtlichen Anspruch eigener Institution und die Unmöglichkeit, eine andere Haltung zu verstehen, die die eigene Glaubenslage überschreitet, bildet eine starke Gewohnheit vieler Christen. Deswegen sollte man auch nach Kardinal Ratzinger sagen, dass es sich lohne, die relative Trennung des Staates von der Kirche, die Gewissensfreiheit, die Menschenrechte und die eigene Verantwortung des Verstandes von der Neuzeit zu übernehmen, mit gleichzeitiger Verwurzelung des Verstandes in Ehrfurcht gegenüber Gott und in

${ }^{16}$ Vgl. E. Stein, Endliches und ewiges Sein. Versuch eines Aufstiegs zum Sinn des Seins, Freiburg im Breisgau 1986, S. 12f.

${ }^{17}$ Vgl. J. Ratzinger, L'intervento del Cardinale Joseph Ratzinger, in: Per una lettura dell'Enciclica FIDES ET RATIO, Città del Vaticano 1999, S. $14 \mathrm{f}$. 
moralischen Grundrechten, die aus dem christlichen Glauben kommen. Denn der Verstand, der sich selbst vergöttert, wird blind. Der heutigen Gesellschaft droht eher das Zurückziehen in die Privatsphäre und die Einfügung in das Pantheon möglicher Wertesysteme. Es widerspricht dem öffentlichen Anspruch des Glaubens an Wahrheit. Kardinal Ratzinger bemerkt hier eine gewisse Aporie, denn indem die Kirche auf den Anspruch auf Wahrheit verzichte, werde sie auch für den Staat nutzlos, wenn jedoch der Staat diesen Anspruch übernehme und unterstütze, sei er nicht mehr pluralistisch. Der künftige Papst sieht eine bestimmte Lösung im Gleichgewicht zwischen diesen beiden Möglichkeiten. Darauf beruhen die Freiheit der Kirche und die Freiheit des Staates. Zur Zeit ist klar, dass der öffentliche Anspruch des Glaubens den Pluralismus und die religiöse Toleranz des Staates nicht beeinflussen sollte. Daraus darf man aber nicht auf eine völlige Neutralität des Staates im Bereich der Werte schließen. Der Staat sollte anerkennen, dass die grundlegende Struktur der christlichen Werte die Bedingung für sein Bestehen ist ${ }^{18}$.

\section{Bibliography}

Chesterton G. K., Wiekuisty Człowiek, Warszawa 2013.

Johannes Paul II, Predigt von Papst Johannes Paul II am 7. Juni 1979 in Auschwitz-Birkenau. Ratzinger J., Demokratie - Pluralismus - Christentum, Leutesdorf 1984.

Ratzinger J., Einführung in das Christentum. Vorlesungen über das Apostolische Glaubensbekenntnis, München 1968.

Ratzinger J., Kirche, Ökumene und Politik, Einsiedeln 1987.

Ratzinger J., L'intervento del Cardinale Joseph Ratzinger, in: Per una lettura dell'Enciclica FIDES ET RATIO, Città del Vaticano 1999.

Ratzinger J., Weggemeinschaft des Glaubens, Augsburg 2002.

Stein E., Endliches und ewiges Sein. Versuch eines Aufstiegs zum Sinn des Seins, Freiburg im Breisgau 1986.

Stein E., Kreuzeswissenschaft. Studie über Johannes vom Kreuz, Freiburg im Breisgau 2003. Stein E., Was ist der Mensch? Eine theologische Anthropologie, Freiburg im Breisgau 1994. Thomas von Aquin, Quaestiones disputatae "De veritate", q. 14.

Wielgus S., Paweł Włodkowic, in: Encyklopedia filozofii polskiej, Band 2, Lublin 2011, S. 306-311.

18 Vgl. J. Ratzinger, Kirche, Ökumene und Politik, Einsiedeln 1987, S. $28 f$. 
Włodkowic Paweł, Pisma wybrane Pawła Włodkowica, ed. L. Ehrlich, Band 1, Instytut Wydawniczy PAX, Warszawa 1968.

Włodkowic Paweł, Pisma wybrane Pawła Włodkowica, ed. L. Ehrlich, Band 2, Instytut Wydawniczy PAX, Warszawa 1966.

Włodkowic Paweł, Pisma wybrane Pawła Włodkowica, ed. L. Ehrlich, Band 3, Instytut Wydawniczy PAX, Warszawa 1969. 